\title{
Laparoscopic splenectomy and esophagogastric devascularization combined with fast-track principles offers greater benefit for patients with portal hypertension
}

\author{
Dong Wang, Zhang Zhang, Rui Dong, Jianguo Lu, Jikai Yin \\ Department of General Surgery, TangDu Hospital, Fourth Military Medical University, Xi'an, Shannxi Province, China
}

Videosurgery Miniinv 2022; 17 (2): 326-337

DOI: https://doi.org/10.5114/wiitm.2021.112680

\begin{abstract}
Introduction: Laparoscopic splenectomy and esophagogastric devascularization (LSED) is becoming increasingly popular in the treatment of esophageal-fundic variceal bleeding with portal hypertension (PHT) in China, and its high safety and minimal trauma have been proven. Fast-track (FT) surgery improves patient recovery and decreases postoperative complications.

Aim: To determine whether LSED with fast-track principles can provide better outcomes than traditional treatment for patients with $\mathrm{PHT}$.

Material and methods: A total of 140 patients who underwent LSED with either traditional treatment or fast-track principles in our department were retrospectively analyzed. The postoperative outcomes, complications, inflammatory mediators, portal vein thrombosis (PVT) and recurrent esophagogastric variceal bleeding rate were recorded. Results: No significant differences were found in the patients' preoperative characteristics. The FT group had better outcomes than the non-FT group with respect to gastrointestinal function recovery, resumption of oral intake, and postoperative hospitalization. The incidence of postoperative complications, including pneumonia, severe ascites, and urinary tract infection, were significantly lower in the FT than the non-FT group. The C-reactive protein and interleukin 6 concentrations and the incidence of PVT were significantly lower in the FT than the non-FT group. The overall recurrent bleeding rate is $11.5 \%$ and no significant difference was found between the two groups in the follow-up period.

Conclusions: LSED with fast-track principles was superior to LSED with traditional treatment in terms of postoperative outcomes, complications, postoperative inflammatory reactions, and the incidence of PVT. This strategy is safe and effective for the treatment of PHT.
\end{abstract}

Key words: laparoscopic splenectomy, devascularization, portal hypertension, fast-track surgery.

\section{Introduction}

Portal hypertension (PHT) is a common clinical syndrome defined by increased pressure in the portal venous system. In China, the main cause of PHT is decompensated cirrhosis due to the hepatitis $B$ virus. PHT usually leads to variceal hemorrhage, splenomegaly, and hypersplenism. Variceal hem- orrhage is associated with a mortality rate ranging from about $7 \%$ to $15 \%$ [1]. Patients who have survived variceal hemorrhage still have a high risk of rebleeding (60\% in the first year) and a mortality rate of up to $33 \%$ [2]. Therefore, surgical treatment for bleeding or prevention of rebleeding is important for recovery of patients. Splenectomy and esophagogas-

\section{Address for correspondence}

Jikai Yin, Department of General Surgery, TangDu Hospital, Fourth Military Medical University, Xi'an, Shannxi Province, China,

e-mail: tdyjk07@fmmu.edu.cn 
tric devascularization (SED), first reported by Yang and Qiu [3] in China, is widely used in China to treat variceal bleeding caused by PHT and has achieved satisfactory results. However, the traditional open procedure is associated with marked tissue trauma and slow postoperative recovery. With the recent technical advances in laparoscopic surgery, laparoscopic SED (LSED) has become a routine procedure for treatment of PHT in many hospitals in China. Notably, LSED is still challenging for many surgeons because of intraoperative bleeding and massive splenomegaly. Fast-track (FT) surgery programs, first described by Bardram et al. [4], have been well developed for management of the immediate perioperative period. The application of fast-track principles greatly accelerates postoperative recovery, reduces the physiologic stress response, lowers the morbidity rate, shortens the hospital stay, and decreases treatment-related costs for surgical patients.

\section{Aim}

Up to now, few studies have focused on LSED combined with fast-track principles for treatment of PHT. In the present study, we examined the safety and efficacy of concomitant LSED and fast-track principles with respect to operative outcomes and complications in the treatment of PHT.

\section{Material and methods}

\section{Patients}

We retrospectively reviewed 145 patients with cirrhotic PHT who underwent LSED in our department from 2016 to 2018. Five patients were excluded due to conversion to open surgery. Finally, 140 patients (92 men and 48 women) who underwent totally LSED were analyzed. All patients showed no intention of liver transplantation after routine preoperative inquiry. Seventy-two patients (47 men and 25 women; mean age: $45.5 \pm 11.1$ years) were treated with a laparoscopic operation in the traditional manner from January 2016 to June 2017. Sixty-eight patients (45 men and 23 women; mean age: 44.2 \pm 12.4 years) were treated with LSED combined with fast-track principles from July 2017 to December 2018. Ninety-two patients had hepatitis B virus-related cirrhosis, 31 had hepatitis $C$ virus-related cirrhosis, 15 had autoimmune hepatitis cirrhosis, and 2 had unexplained cirrhosis. Fifty-four patients had
Child-Pugh class A liver disease and 86 had ChildPugh class B. Patients with Child-Pugh class $C$ liver disease were excluded because of their poor recovery and high mortality. The indocyanine green 15 -minute retention rate was measured for assessment of liver function. A history of variceal hemorrhage (hematemesis and melena) was an absolute indication for a laparoscopic operation. Surgery was also indicated for patients with no history but a high risk of variceal hemorrhage; simultaneous grade III esophageal varices, blue varices, or cherry red spots from bleeding varices diagnosed by endoscopy [5-7]; hypersplenism; and a high hepatic venous pressure gradient of $>12 \mathrm{~mm} \mathrm{Hg}$ [8].

The study protocol was approved by the ethics committee of TangDu Hospital, and informed consent was obtained from each patient. All procedures were performed in accordance with the relevant guidelines and regulations.

\section{Operative design and procedure}

To ensure the most individualized operation and precise medical treatment possible, all patients underwent preoperative multidetector-row computed tomography to evaluate the splenic volume and determine the location of the splenic artery, left gastric vein, and portosystemic collateral vessels. For the splenectomy procedure, each patient was placed in the supine position with the left flank elevated at a 35-degree angle. The locations of the trocars are shown in Photo 1 A. A 12-mm laparoscopic trocar was inserted through an incision beneath the umbilicus (point $b$ in Photo $1 \mathrm{~A}$ ). Carbon dioxide pneumoperitoneum was established using a high-flow electric insufflator, and four other trocars were inserted under visual control: two 12-mm laparoscopic trocars were separately inserted through the crossover point of the right axillary midline and navel horizontal line (point a in Photo $1 \mathrm{~A}$ ) and at the level of the lower pole of the spleen (point $c$ in Photo $1 \mathrm{~A}$ ), and two 5-mm laparoscopic trocars were inserted at a point along the ventrimeson $3 \mathrm{~cm}$ below the xiphoid process and through the midpoint between the first location of the 5-mm laparoscopic trocar and the navel (points e and $d$ in Photo $1 \mathrm{~A}$ ). Point a was used as the laparoscopic observation incision, Points $b$ and $c$ were used as the main operative incisions, and Points $d$ and e were used as the assistant operative incisions. Electrocautery, the LigaSure 

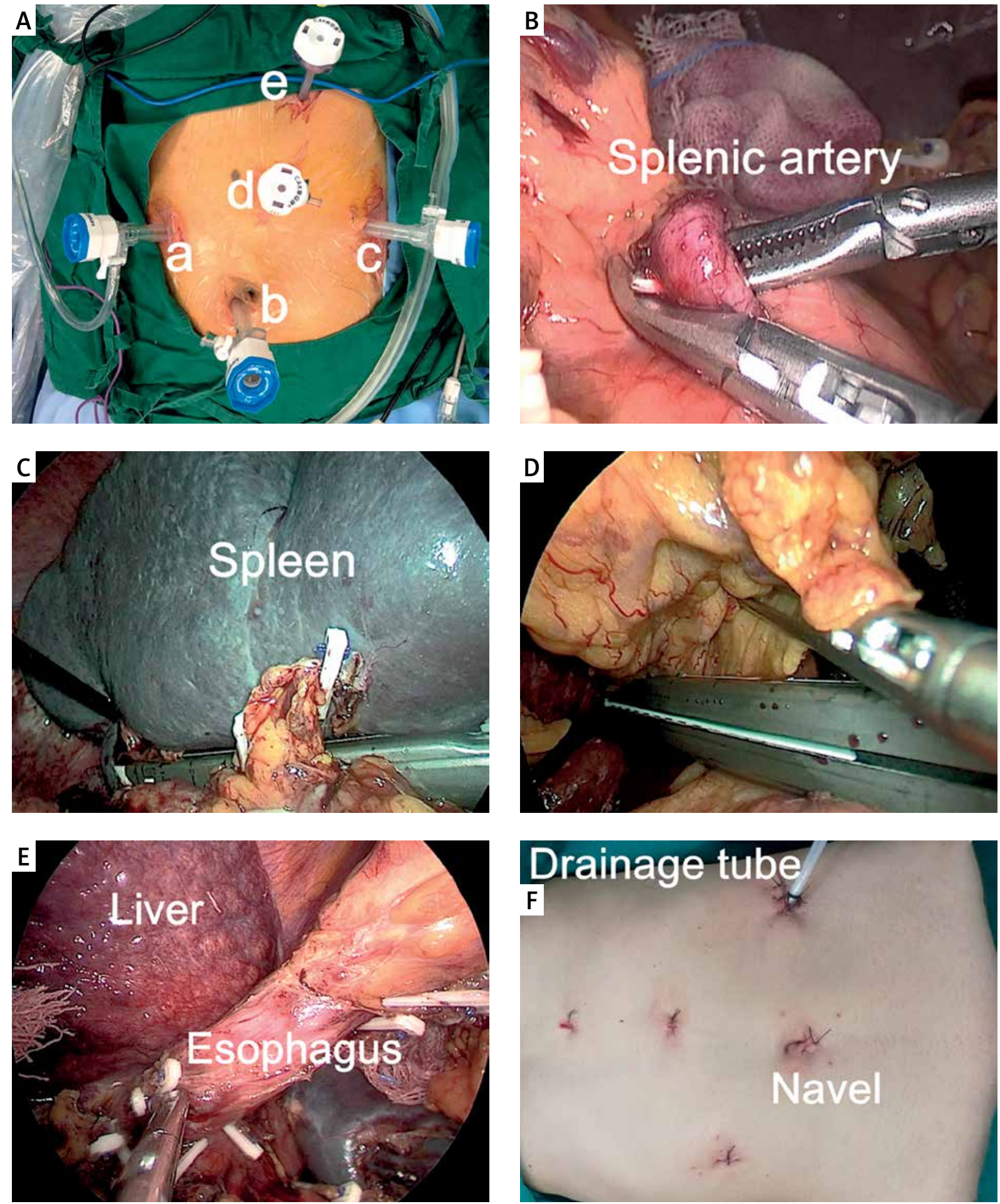

Photo 1. Locations of trocars and key steps in laparoscopic splenectomy and esophagogastric devascularization. A - Trocar locations. (a) Laparoscopic observation incision (below or to the right of the navel). (b, c) Main operative incisions. (d, e) Assistant operative incisions. B - Separation and ligation of the splenic artery. C - Transection of splenic hilar pedicles with the Endo GIA stapler. D - Transection of the left gastric vein at the root with the Endo GIA stapler. E - Esophagogastric devascularization. F - Completion of totally laparoscopic surgery 
vessel-sealing system (Medtronic, Minneapolis, MN, USA), hemostatic clamps, and the Endo GIA stapler (Medtronic) were used for vessel disruption.

In most patients, after opening the omental bursa, the splenic artery was separated and ligated (Photo 1 B). Without a blood supply, the spleen volume decreased, making splenic dissection easier to perform and decreasing splenic hemorrhage during the operation. The spleen was transected with an Endo GIA stapler (60-2.5 mm) after all visible splenic attachments had been dissected (Photo $1 \mathrm{C}$ ). After dissociating and cutting the left gastric vein at the root with an Endo GIA stapler (45-2.5 mm) (Photo $1 \mathrm{D})$, the soft tissues and variceal veins were dissected along the greater curvature and lesser curvature of the stomach. The varices along the stomach and lower part of the esophagus (an approximately 5 -cm segment) were also resected (Photo $1 \mathrm{E}$ ). The spleen was placed in a specimen bag in the abdominal cavity, fragmented within the specimen bag, and removed from the incision in the left lower quadrant (elongated to a $3-\mathrm{cm}$ incision). A drainage tube was placed in the left upper abdomen. The LSED procedure was thus completed (Photo 1 F).

\section{Perioperative management in FT group and non-FT group}

The FT group was managed perioperatively according to the standard fast-track principles. The patients and their families were given detailed information on the perioperative treatment and rehabilitation programs to increase their compliance. Oral carbohydrates were administered in the early morning of the operative day. During the procedure, the patient's body was kept warm, and warm normal saline was used to wash the abdominal cavity. The infused fluid, especially the crystalloid solution, was controlled to reduce the cardiac burden and tissue interstitial edema. Patient-controlled analgesia was administered by continuous perfusion through a peripheral vein within $48 \mathrm{~h}$ postoperatively. The Foley catheter was removed on the morning of the first postoperative day. Gastric decompression was stopped within the first $12 \mathrm{~h}$ after surgery. The abdominal drain was removed after $48 \mathrm{~h}$ when no further bloody fluid was observed and the amylase concentration of the abdominal drainage fluid was less than three times the serum concentration. The patients were encouraged to sit and engage in in-bed activities the day after the operation, and out-of-bed activities were allowed after the first postoperative day. The patients were encouraged to drink small amounts of water after recovery from anesthesia. When flatus and oral tolerance were reached, a semi-liquid to soft-food diet was gradually introduced. Enoxaparin (0.4 IU twice daily) was subcutaneously injected on postoperative day 2 if no obvious bloody abdominal drainage was observed and the peripheral red blood cell count was stable, which decreased the risk of portal vein thrombosis (PVT) formation. The patients received respiratory physiotherapy and atomizing inhalation of ambroxol during the first $72 \mathrm{~h}$ postoperatively. Supplemental plasma or human albumin was administered to maintain the serum albumin concentration at $\geq 30 \mathrm{~g} / \mathrm{l}$. A balance was maintained between the liquid intake and output, and furosemide $(20 \mathrm{mg} /$ day) was intravenously injected during the first $72 \mathrm{~h}$ postoperatively. Somatostatin was used during the first $48 \mathrm{~h}$ postoperatively.

Patients in the non-FT group were treated in the traditional manner. They were provided conventional information on PHT. All patients in this group underwent general anesthesia, and a gastric drainage tube, intra-abdominal drain, and urinary catheter were routinely inserted. Postoperative oral intake and rehabilitation were performed in the traditional manner. The details of the perioperative treatments are shown in Table I.

\section{Operative outcomes and complications}

The operative outcomes were the operation time, blood loss volume, postoperative hospital stay, and postoperative mortality and morbidity rates. Any death that occurred in the hospital after the operation was used to calculate the mortality rate. The time to first flatus, first bowel movement, and oral intake and the postoperative hospital stay were recorded. Postoperative complications, including intra-abdominal hemorrhage, gastrointestinal fistula, wound infection, pneumonia, and urinary tract infection, were also assessed.

\section{PVT assessment}

A Doppler ultrasound examination of the portal vein was performed preoperatively and postoperatively. The postoperative examination was performed 3,7 , and 14 days after the surgery. PVT was defined as complete or partial (>50\%) occlusion of the portal vein trunk, superior mesenteric vein, or splenic vein. A peripheral venous blood sample was collected from 
Table I. Peri-operative management compared between the two groups

\begin{tabular}{|c|c|c|}
\hline & FT group & Non-FT group \\
\hline \multirow{4}{*}{$\begin{array}{l}\text { Pre- } \\
\text { operative }\end{array}$} & Detailed preoperative consultation and education & Similar education without fast-track surgery principles \\
\hline & $\begin{array}{c}\text { Solid food and liquid fasting for } 6 \text { and } 2 \mathrm{~h} \text {, respectively } \\
\text { Oral carbohydrates were administered in the early } \\
\text { morning of the operative day }\end{array}$ & Fast from the night before operation \\
\hline & No preoperative use of nasogastric tube & Routine use of nasogastric tube \\
\hline & No intestinal preparation & Routine intestinal preparation \\
\hline \multirow[t]{4}{*}{$\begin{array}{l}\text { Intra- } \\
\text { operative }\end{array}$} & $\begin{array}{l}\text { Anesthesia: limit infusions; shorten anesthesia time; } \\
\text { avoid hypothermic conditions }\end{array}$ & $\begin{array}{l}\text { Anesthesia: traditional way without special require- } \\
\text { ments }\end{array}$ \\
\hline & use nasogastric tube after anesthesia & \\
\hline & Surgical operation: operative time control & Conventional operation \\
\hline & Routine surgical drainage tubes & Routine surgical drainage tubes \\
\hline \multirow[t]{3}{*}{$\begin{array}{l}\text { Post- } \\
\text { operative }\end{array}$} & $\begin{array}{l}\text { Early mobilization: in-bed activities the day after the } \\
\text { operation, out of bed activities on the } 1^{\text {st }} \text { postopera- } \\
\text { tive day, then gradually increasing exercise to resume } \\
\text { normal ambulation }\end{array}$ & Early mobilization: no \\
\hline & $\begin{array}{l}\text { Enteral nutrition: drink small amounts of water after } \\
\text { recovery from anesthesia; take liquid diet on the } 1^{\text {st }} \\
\text { postoperative day, then gradually introduce a semi-liq- } \\
\quad \text { uid to soft-food diet; limit intravenous infusions }\end{array}$ & Enteral nutrition: no \\
\hline & $\begin{array}{l}\text { Remove urine catheter on the morning of postoperative } \\
\text { day 1; remove gastric tube within the first } 12 \mathrm{~h} \text { postop- } \\
\text { eratively; remove the abdominal drain after } 48 \mathrm{~h} \\
\text { Anticoagulation began on postoperative day } 2 \text { if no } \\
\text { abdominal bleeding }\end{array}$ & $\begin{array}{l}\text { Remove urine catheter on postoperative day } 2-3 \text {; } \\
\text { remove gastric tube after the first flatus; remove the } \\
\text { abdominal drain 1-2 day before discharge } \\
\text { Anticoagulation: no }\end{array}$ \\
\hline
\end{tabular}

each patient on preoperative day 1 and postoperative days $1,3,7$, and 14 . The D-dimer concentration and platelet count were also determined.

\section{Measurement of inflammatory mediators}

The serum levels of C-reactive protein (CRP) and interleukin 6 (IL-6), well-known inflammatory indicators, were detected by enzyme-linked immunosorbent assay kits on preoperative day 1 and postoperative days 1,3 , and 7 .

\section{Postoperative follow-up}

Long-term follow-up was performed by telephone or at the outpatient department.

\section{Statistical analysis}

Continuous data are expressed as mean \pm standard deviation and were analyzed by the $t$-test. Categorical data are expressed as number and percentage and were analyzed by the $\chi^{2}$ test. A $p$-value of
$<0.05$ was considered statistically significant. All statistical analyses were performed using SPSS Version 25 (IBM Corp., Armonk, NY, USA).

\section{Results}

\section{Patients' characteristics}

The patients' basic characteristics are shown in Table II. The conversion rate to an open procedure was $3.4 \%$ (5/145), 3 of which were due to intraoperative bleeding and 2 were due to abdominal adhesion. No significant difference was found in age, sex, $\mathrm{BMI}$, etiology, indocyanine green 15-minute retention rate, Child-Pugh class, history of variceal hemorrhage, hepatic venous pressure gradient, routine blood examination findings, or serum biochemical parameters between the two groups.

\section{Intraoperative and postoperative data}

There was no significant difference in the operative time or blood loss volume between the FT and 
Table II. Characteristics of patients with portal hypertension

\begin{tabular}{|c|c|c|c|}
\hline Factors & FT group & Non-FT group & $P$-value \\
\hline \multicolumn{4}{|l|}{ Sex: } \\
\hline Male & 45 & 47 & 0.911 \\
\hline Female & 23 & 25 & \\
\hline Age & $44.2 \pm 12.4$ & $45.5 \pm 11.1$ & 0.514 \\
\hline \multicolumn{4}{|l|}{ Etiology: } \\
\hline HBV & 45 & 47 & \\
\hline $\mathrm{HCV}$ & 15 & 16 & \\
\hline Autoimmune hepatitis & 7 & 8 & \\
\hline Others & 1 & 1 & \\
\hline BMI $\left[\mathrm{kg} / \mathrm{m}^{2}\right]$ & $23.8 \pm 5.9$ & $23.3 \pm 5.2$ & 0.595 \\
\hline Longitudinal diameter of spleen [mm] & $171.6 \pm 34.4$ & $163.4 \pm 32.2$ & 0.205 \\
\hline ICG R15 \% & $21.2 \pm 13.4$ & $20.5 \pm 14.6$ & 0.768 \\
\hline Child-Pugh & $7.03 \pm 1.26$ & $7.04 \pm 1.34$ & 0.956 \\
\hline Class A & 26 & 28 & \\
\hline Class B & 42 & 44 & \\
\hline $\mathrm{HVPG}[\mathrm{mm} \mathrm{Hg}]$ & $18.2 \pm 7.2$ & $17.2 \pm 6.7$ & 0.396 \\
\hline Variceal hemorrhage history: & & & 0.902 \\
\hline Yes & 46 & 48 & \\
\hline No & 22 & 24 & \\
\hline White blood cells (WBC) [× 109/l] & $2.1 \pm 1.9$ & $2.3 \pm 1.7$ & 0.512 \\
\hline Hemoglobin [g/dl] & $98.6 \pm 30.3$ & $104.4 \pm 29.8$ & 0.256 \\
\hline Platelet count $\left[\times 10^{9} / \mathrm{ml}\right]$ & $53.3 \pm 25.5$ & $48.4 \pm 26.1$ & 0.245 \\
\hline D-dimer [ $\mathrm{gg} / \mathrm{ml}]$ & $1.3 \pm 0.5$ & $1.2 \pm 0.4$ & 0.192 \\
\hline Total bilirubin [mmol/l] & $24.9 \pm 12.7$ & $23.2 \pm 9.8$ & 0.375 \\
\hline Prothrombin time [s] & $15.8 \pm 2.4$ & $16.2 \pm 2.2$ & 0.305 \\
\hline Prothrombin activity (PTA, \%) & $65.2 \pm 10.4$ & $67.3 \pm 11.6$ & 0.262 \\
\hline
\end{tabular}

$B M I$ - body mass index, ICG R15 - Indocyanine green 15-minute retention rate, HVPG - hepatic venous pressure gradient. All of the values represent the mean $\pm S D$ (standard deviation)

non-FT groups $(p>0.05)$. According to the fast-track principles, the abdominal drain was removed much earlier in the FT than the non-FT group $(p<0.05)$. Patients in the FT group had their first bowel movement much earlier than those in the non-FT group $(p<0.05)$. Similarly, the time to first postoperative flatus was significantly shorter in the FT than the non-FT group $(p<0.05)$. Patients in the FT group were encouraged to drink and eat earlier. Therefore, under fast-track guidance, the patients in the FT group were discharged from the hospital significantly earlier than those in the non-FT group $(p<0.05)$ (Table III).

\section{Postoperative complications}

Within 30 days postoperatively, one patient in the FT group died of severe intraperitoneal hemorrhage and 1 patient in the non-FT group died of abdominal infection caused by a gastric fistula. The incidence of infectious complications including pneumonia, severe ascites, and urinary tract infection was significantly higher in the non-FT than the FT group $(p<0.05)$. However, the incidence rates of incision site infection and intra-abdominal infection were not significantly different between the groups. Similarly, 
Table III. Intra- and post-operative data

\begin{tabular}{|lccc|}
\hline Parameter & FT group & Non-FT group & $P$-value \\
\hline Operation time [min] & $242.4 \pm 67.3$ & $238.5 \pm 78.4$ & 0.753 \\
\hline Blood loss [ml] & $283.8 \pm 134.2$ & $296.2 \pm 118.4$ & 0.563 \\
\hline Abdominal drain [days] & $3.2 \pm 1.6$ & $5.4 \pm 2.1$ & $<0.001$ \\
\hline First flatus [days] & $2.4 \pm 1.4$ & $3.1 \pm 1.6$ & 0.007 \\
\hline First defecation [days] & $3.9 \pm 1.4$ & $5.1 \pm 2.1$ & $<0.001$ \\
\hline Taking liquid food [days] & $4.5 \pm 1.8$ & $6.2 \pm 2.3$ & $<0.001$ \\
\hline Postoperative hospital stay [days] & $6.3 \pm 2.3$ & $8.4 \pm 2.2$ & 0.001 \\
\hline
\end{tabular}

the incidence of surgical complications (abdominal bleeding, encephalopathy, pancreatic fistula, and liver failure) was not significantly different between the two groups (Table IV).

\section{Inflammatory indicators}

The levels of inflammatory indicators (CRP and IL-6) were not significantly different between the two groups on preoperative day 1 . The postoperative CRP and IL-6 levels in both groups were markedly higher than those preoperatively. However, the levels of CRP and IL- 6 on postoperative days 1, 3, and 7 were significantly lower in the FT than the non-FT group $(p<0.05)$ (Figure $1 \mathrm{~A})$.

\section{PVT}

As shown in Table IV, although 10 of 68 (14.7\%) patients in the FT group and 12 of 72 patients $(16.7 \%)$ in the non-FT group were diagnosed with PVT by preoperative Doppler ultrasonography and computed tomography examination, the $\chi^{2}$ test showed no significant difference between the two groups ( $p=0.875)$. However, 7 of 58 patients (13.8\%) in the FT group and 17 of 60 patients (28.3\%) in the non-FT group developed PVT after the operation. The incidence of PVT was significantly lower in the FT than the non-FT group postoperatively $(p=0.028)$. The D-dimer level was much lower in the FT than the non-FT group on postoperative days 3, 7, and 14

Table IV. Postoperative complications and follow-up data

\begin{tabular}{|c|c|c|c|}
\hline Parameter & FT group & Non-FT group & $P$-value \\
\hline Emergency operation for bleeding, $n$ & 2 & 2 & 1 \\
\hline Encephalopathy, $n$ & 0 & 0 & 1 \\
\hline Mortality, $n$ & 1 & 1 & 1 \\
\hline Incision site infection, $n$ & 1 & 2 & 0.441 \\
\hline Pancreatic fistula, $n$ & 1 & 1 & 1 \\
\hline Pneumonia, $n$ & 1 & 9 & 0.018 \\
\hline Hemorrhage requiring transfusion, $n$ & 3 & 4 & 1 \\
\hline Postoperative liver failure, $n$ & 0 & 0 & 1 \\
\hline Severe ascites, $n$ & 2 & 10 & 0.032 \\
\hline Urinary tract infection, $n$ & 1 & 8 & 0.034 \\
\hline Intra-abdominal infection, $n$ & 1 & 1 & 1 \\
\hline Follow-up data $(n=122)$ & 62 & 60 & \\
\hline Recurrent esophagogastric bleeding, $n$ (\%) & $7(11.3 \%)$ & $7(11.7 \%)$ & 0.948 \\
\hline Death & 0 & 0 & \\
\hline Receive liver transplantation & 0 & 0 & \\
\hline
\end{tabular}


A

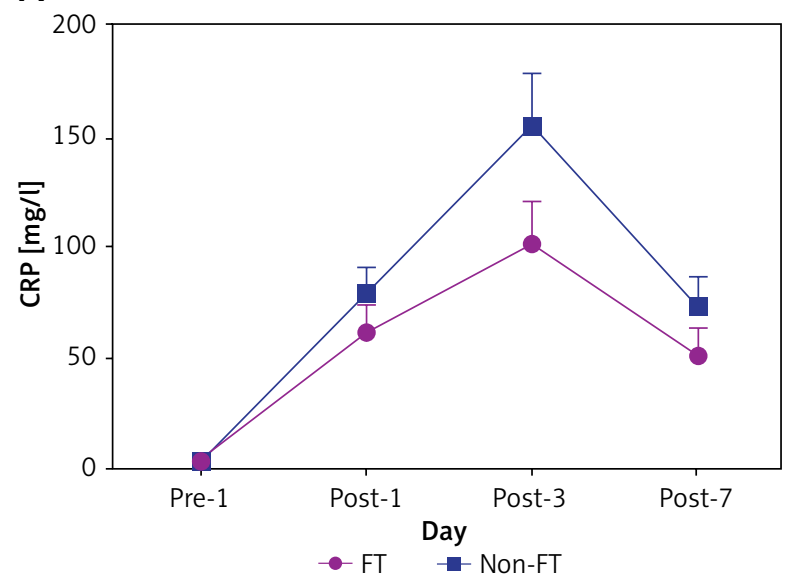

B

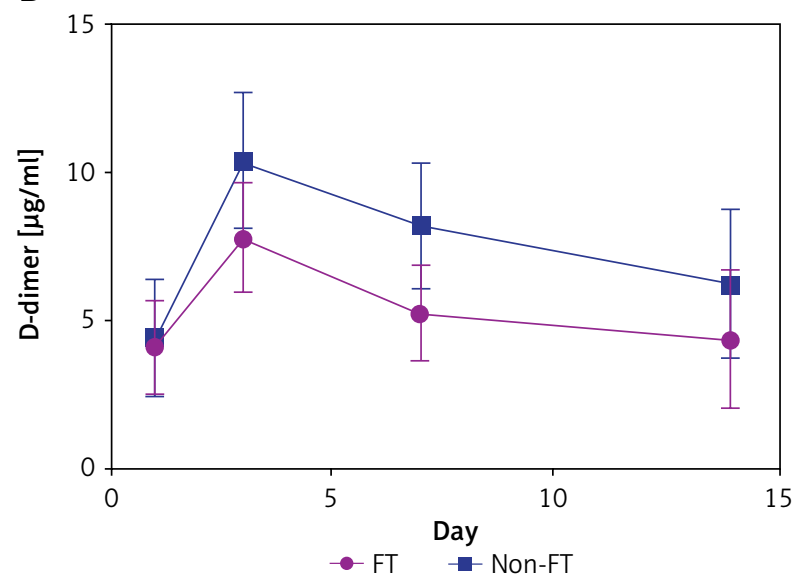

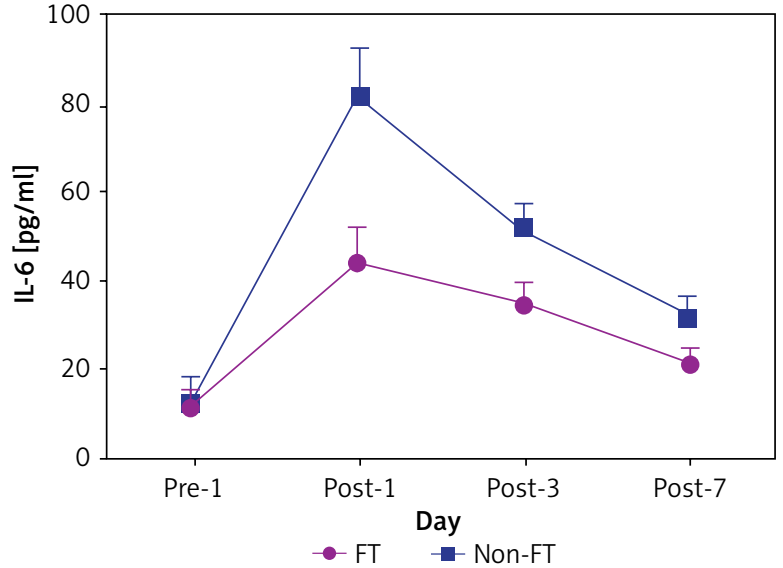

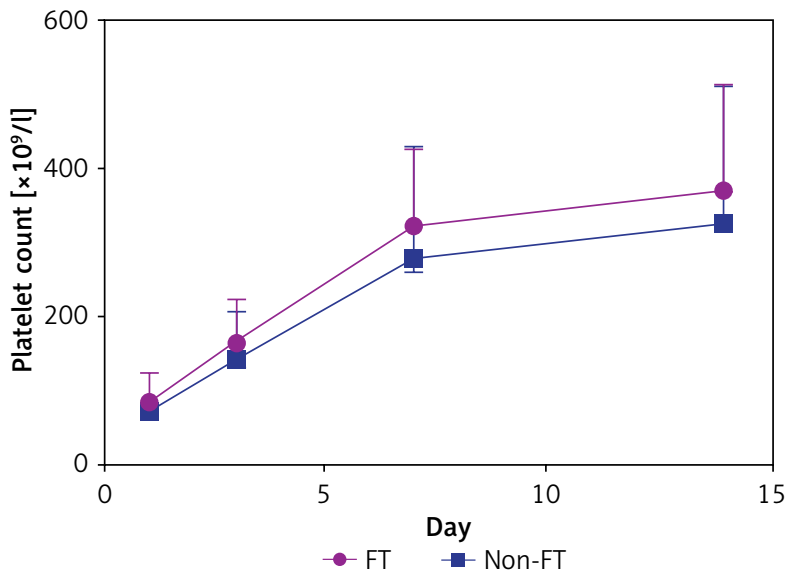

Figure 1. A - Changes in the CRP and IL-6 levels preoperatively and postoperatively. B - Changes in D-dimer level and platelet count postoperatively

Pre-preoperatively, Post-postoperatively.

$(p<0.05)$. The platelet count was significantly higher in the FT than the non-FT group on postoperative days 3, 7, and 14 (Table $V$ and Figure $1 \mathrm{~B}$ ).

\section{Follow-up}

Sixty-two out of the 71 patients (one died after operation was excluded, $87.3 \%$ ) in the nonFT group were followed up with a mean duration of 19.1 months (1-43 months). In the FT group, the follow-up rate was $89.6 \%$ (1 died after operation was excluded, 60/67) and the mean follow-up time was 17.8 months (1.2-28 months). All the patients in both groups are alive. No patient received liver transplantation. During the follow-up period, $7(11.3 \%)$ patients in the non-FT group and 7 in the FT group (11.7\%) experienced recurrent esophagogastric variceal hemorrhage. The overall variceal re- bleeding rate was $11.5 \%$. No significant difference was found between the two groups ( $p=0.948)$ (Table IV). The esophagogastric variceal rebleeding was controlled by drug, endoscopy or TIPS treatment.

\section{Discussion}

For many decades, SED has been widely performed in China and Japan because of its effective control of variceal bleeding, variceal rebleeding, and secondary hypersplenism with little impairment of liver function and a low incidence of encephalopathy $[9,10]$. Variceal hemorrhage is currently the major indication for treatment of PHT. Although non-cardioselective $\beta$-blockers and variceal band ligation have been recommended for primary prophylaxis of variceal hemorrhage in patients with cirrhosis [11, 12], splenomegaly and hypersplenism cannot be 
Table V. Portal vein thrombosis

\begin{tabular}{|lccc|}
\hline Parameter & FT group & Non-FT group & $P$-value \\
\hline Portal vein thrombosis: & $10(14.7 \%)$ & $12(16.70 \%)$ & 0.75 \\
\hline Before operation & $7 / 58(13.8 \%)$ & $17 / 60(28.3 \%)$ & 0.028 \\
\hline After operation & & & 0.344 \\
\hline D-dimer $[\mathrm{\mu g} / \mathrm{ml}]:$ & $4.1 \pm 1.5$ & $4.4 \pm 1.9$ & $<0.001$ \\
\hline Post-1 & $7.8 \pm 1.8$ & $10.4 \pm 2.3$ & $<0.001$ \\
\hline Post-3 & $5.2 \pm 1.6$ & $8.2 \pm 2.1$ & $<0.001$ \\
\hline Post-7 & $4.3 \pm 2.3$ & $6.2 \pm 2.5$ & 0.058 \\
\hline Post-14 & & $72.4 \pm 46.8$ & 0.009 \\
\hline Platelet count $\left[\times 10^{9} / 1\right]:$ & $84.2 \pm 37.6$ & $141.8 \pm 64.6$ & 0.02 \\
\hline Post-1 & $164.8 \pm 54.4$ & $278.2 \pm 148.7$ & 0.083 \\
\hline Post-3 & $321.8 \pm 102.6$ & $327.4 \pm 182.2$ & \\
\hline Post-7 & $369.2 \pm 142.7$ & & \\
\hline Post-14 & & & \\
\hline
\end{tabular}

resolved using this technique. Studies have shown that splenectomy can reduce portal venous pressure and improve liver function [13-15]. SED can not only reduce the high risk of variceal bleeding but also lessen the adverse effects of splenomegaly and hypersplenism. LSED has obvious advantages over SED in terms of less trauma and faster recovery [16-18]. Therefore, LSED is an important treatment for patients with a high risk of variceal hemorrhage in our department. The LSED procedure, first applied in our department in 2011, became the first-line therapy for PHT in 2014 after we had accumulated 3 years of clinical experience. The safety and effectiveness of LSED have been confirmed by our own clinical experience and other researchers [10, 19]. Although a limitation of the present study is our retrospective analysis of patients at two different stages, no significant differences in the intraoperative data were found between the two groups, indicating that the operator's experience and laparoscopic technique were not the beneficial factors in the FT group. Fasttrack principles have been extensively used in clinical perioperative management. The widely acknowledged advantages of fast-track principles include acceleration of postoperative recovery, reduction of perioperative morbidity, and shortening of the hospital stay. Most patients with $\mathrm{PHT}$ requiring surgery had liver cirrhosis for many years and are in a poor general condition; they often exhibit decompensated liver function, hypoalbuminemia, thrombocytopenia, and coagulation disorders. Less surgical trauma and more rapid recovery from surgery appear particularly important for these patients. Therefore, LSED combined with fast-track principles may be a suitable treatment method. Research has shown that fast-track principles reduce the hospital stay from 8.4 to 6.3 days on average. Significantly improved postoperative recovery with respect to toleration of a liquid diet, first flatus, and first defecation has been observed. The incidence of pneumonia, urinary tract infection, and the inflammatory response is significantly reduced with fast-track principles, and a decreased incidence of PVT has been detected.

Gastrointestinal dysfunction frequently occurs in postoperative patients and can intensify the therapeutic efforts and prolong the hospital stay [20-22]. Many Chinese people have long held a misconception that good recovery requires long-term bed rest. Operation-related stress is also not conducive to postoperative recovery. Thus, high-quality preoperative education is necessary to rectify inaccuracies and relieve patients' anxieties. When sufficient and proper preoperative education is provided, patients' cooperation and motivation increase [23]. Pain management is also very important in the FT protocol. Proper postoperative analgesia can not only decrease the pain-related stress response but can also eliminate the discomfort of early ambulation [24]. Opioids are a commonly used analgesic after surgery, but they may delay gastrointestinal mobility and even cause nausea and vomiting, which may slow recovery [25]. Because LSED is a minimally inva- 
sive approach that induces less pain than traditional open surgery, postoperative analgesia was administered in this study only when the pain could not be tolerated. Therefore, with the help of comprehensive treatment guidance, patients' compliance with outof-bed activities at an early stage was significantly improved in the FT group. Beyond that, placement of a long-term indwelling gastric tube is a risk factor for nausea and vomiting. In many operations, a nasogastric tube is not yet routinely placed in accordance with the fast-track policy. However, SED may lead to ischemia of the gastric fundus, resulting in a gastric fistula. Therefore, we routinely placed a nasogastric feeding tube to relieve intragastric pressure but terminated it within the first $12 \mathrm{~h}$ postoperatively in the FT group. With sufficient preoperative education, adequate pain management, and early mobilization, the patients in the FT group showed earlier return of intestinal function postoperatively.

Many studies have shown that the fast-track protocol has positive effects on the human immune system and decreases the perioperative stress response to surgical trauma [26]. The normal inflammatory response, first proposed by Cuthbertson and Tilstone [27], may play a vital role in the process of wound healing and infection resistance; however, it may also lead to pain, emergence of opportunistic infections, fatigue, and organ dysfunction. Multiple factors determine the severity of the postoperative inflammatory reaction. Early recovery of gastrointestinal activity maintains the intestinal mucosal barrier function and can help to maintain the immune function. Compared with open surgery, laparoscopic minimally invasive surgery and less operative bleeding are both beneficial for reduction of the inflammatory reaction. Proinflammatory cytokines such as CRP and IL-6, which are triggered by the inflammatory reaction [28], are commonly used as inflammatory indicators. IL- 6 is reportedly associated with the severity of surgical trauma [29]. IL-6 can also stimulate the liver to synthesize CRP and enhance the inflammatory response [30]. In the present study, we found no significant difference in the preoperative levels of inflammatory indicators between the two groups. Both the IL- 6 and CRP levels increased significantly after the operation. However, the IL-6 and CRP levels were significantly lower in the FT than the non-FT group on postoperative days 1,3 , and 7 ( $p<$ $0.05)$. All patients in both groups underwent totally laparoscopic surgery. No statistically significant dif- ference was found in the operation time or intraoperative blood loss volume between the two groups. These findings indicate that rapid rehabilitation may play an important role in reducing the postoperative inflammatory reaction.

PVT is a common and important complication of $\mathrm{PHT}$, especially in patients undergoing splenectomy and azygoportal disconnection. One study showed that the incidence of PVT after laparoscopic surgery was higher than that after open surgery, although the rate of occlusion of the main trunk of the portal vein was similar [31]. PVT may lead to liver dysfunction, ascites, intestinal edema, and an increased risk of variceal bleeding [32]. Therefore, reducing the incidence of PVT is conducive to reducing postoperative complications. The safety of anticoagulation in patients with PHT, especially perioperatively, has been debated for many years because of the risk of bleeding. However, increasingly more studies have shown the safety and effectiveness of anticoagulation in patients with PHT $[33,34]$. In our clinical experience, laparoscopic surgery with meticulous manipulation and adequate intraoperative hemostasis is important for postoperative anticoagulation. In the present study, 3 of 58 patients (5.17\%) in the FT group stopped anticoagulation therapy because of abdominal bleeding (data not shown). Although two patients in the FT group needed emergency surgery, the operation confirmed that the abdominal bleeding had been caused by dropping of a hemostatic clip and poorly executed suturing of the subumbilical trocar incision (the periumbilical abdominal veins are enlarged in patients with $\mathrm{PHT}$ ). In the remaining patient ( 1 of 58 patients, $1.72 \%$ ), the abdominal hemorrhage was stopped after termination of the anticoagulation therapy. Therefore, our study demonstrated the safety of anticoagulation therapy. Additionally, the incidence of PVT in the FT group significantly decreased with anticoagulation therapy. A reduced incidence of PVT may contribute to reductions in gastrointestinal edema, gastrointestinal bacterial translocation, and ascites, thereby promoting gastrointestinal function recovery and lessening postoperative inflammation.

Infection is a risk factor for postoperative morbidity and mortality [35]. Studies have shown that the incidence of infection decreases with use of the FT protocol [36]. In our study, the rates of urethral infection and pneumonia were significantly reduced in the FT group, but no significant difference in the incidence of abdominal or incision infection was 
found; this might have been due to the minimal invasiveness of laparoscopic surgery.

\section{Conclusions}

With the implementation of fast-track concepts for LSED, patients can be discharged from the hospital earlier with fewer complications and an overall higher level of satisfaction. LSED combined with fast-track principles is a safe, minimally invasive protocol for treatment of PHT.

\section{Acknowledgments}

The paper is supported by the National Natural Science Foundation of China (81700533), the Key Research and Development Program of Shaanxi Province, China (2017SF-116, 2015JM8420, 2015KTCL03-05) and the Innovation and Development Foundation of Tangdu Hospital (2017LCYJ003, 2018JSYJ010, 2019LCYJ005).

\section{Conflict of interest}

The authors declare no conflict of interest.

\section{References}

1. Simonetto DA, Liu M, Kamath PS. Portal Hypertension and related complications: diagnosis and management. Mayo Clin Proc 2019; 94: 714-26.

2. Garcia-Tsao G, Bosch J. Varices and variceal hemorrhage in cirrhosis: a new view of an old problem. Clin Gastroenterol Hepatol 2015; 13: 2109-17.

3. Zhen Y, Fazu Q. Pericardial devascularization with splenectomy for the treatment of portal hypertension. Chin J Surg 2000; 38: 645-8.

4. Bardram L, Funch-Jensen P, Jensen P, et al. Recovery after laparoscopic colonic surgery with epidural analgesia, and early oral nutrition and mobilisation. Lancet 1995; 345: 763-4.

5. Palmer ED, Brick IB. Correlation between the severity of esophageal varices in portal cirrhosis and their propensity toward hemorrhage. Gastroenterology 1956; 30: 85-90.

6. Lebrec D, De FP, Rueff B, et al. Portal hypertension, size of esophageal varices, and risk of gastrointestinal bleeding in alcoholic cirrhosis. Gastroenterology 1980; 79: 1139-44.

7. Beppu K, Inokuchi K, Koyanagi N, et al. Prediction of variceal hemorrhage by esophageal endoscopy. Gastrointest Endosc 1981; 27: 213-8.

8. Jalan R, Hayes PC. UK guidelines on the management of variceal haemorrhage in cirrhotic patients. Gut 2000; 46 Suppl 3-4: I1.

9. Zheng S, Sun P, Liu X, et al. Efficacy and safety of laparoscopic splenectomy and esophagogastric devascularization for portal hypertension: a single-center experience. Medicine 2018; 97 : e13703.
10. Kawanaka H, Akahoshi T, Kinjo N, et al. Laparoscopic splenectomy with technical standardization and selection criteria for standard or hand-assisted approach in 390 patients with liver cirrhosis and portal hypertension. J Am Coll Surg 2015; 221: 354-66.

11. Tripathi D, Stanley AJ, Hayes PC, et al. U.K. guidelines on the management of variceal haemorrhage in cirrhotic patients. Gut 2015; 64: 1680-704.

12. Villanueva C, Albillos A, Genesca J, et al. Beta blockers to prevent decompensation of cirrhosis in patients with clinically significant portal hypertension (PREDESCI): a randomised, double-blind, placebo-controlled, multicentre trial. Lancet 2019; 393: 1597-608.

13. Yamamoto N, Okano K, Oshima M, et al. Laparoscopic splenectomy for patients with liver cirrhosis: improvement of liver function in patients with Child-Pugh class B. Surgery 2015; 158: 1538-44.

14. Leite LA, Pimenta FA, Ferreira RC, et al. Splenectomy improves hemostatic and liver functions in hepatosplenic schistosomiasis Mansoni. PLoS One 2015; 10: e135370.

15. Yada A, limuro $\mathrm{Y}$, Uyama $\mathrm{N}$, et al. Splenectomy attenuates murine liver fibrosis with hypersplenism stimulating hepatic accumulation of Ly-6C(lo) macrophages. J Hepatol 2015; 63: 905-16.

16. Cai Y, Liu Z, Liu X. Laparoscopic versus open splenectomy for portal hypertension: a systematic review of comparative studies. Surg Innov 2014; 21: 442-7.

17. Jiang GQ, Chen P, Qian J, et al. Perioperative advantages of modified laparoscopic vs open splenectomy and azygoportal disconnection. World J Gastroenterol 2014; 20: 9146-53.

18. Rodriguez-Luna MR, Balague C, Fernandez-Ananin S, et al. Outcomes of laparoscopic splenectomy for treatment of splenomegaly: a systematic review and meta-analysis. World J Surg 2021; 45: 465-79.

19. Yu H, Guo S, Wang L, et al. Laparoscopic splenectomy and esophagogastric devascularization for liver cirrhosis and portal hypertension is a safe, effective, and minimally invasive operation. J Laparoendosc Adv Surg Tech A 2016; 26: 524-30.

20. Vignali A, Elmore U, Cossu A, et al. Enhanced recovery after surgery (ERAS) pathway vs traditional care in laparoscopic rectal resection: a single-center experience. Tech Coloproctol 2016; 20: 559-66.

21. Willcutts KF, Chung MC, Erenberg CL, et al. Early oral feeding as compared with traditional timing of oral feeding after upper gastrointestinal surgery: a systematic review and meta-analysis. Ann Surg 2016; 264: 54-63.

22. Pedziwiatr M, Mavrikis J, Witowski J, et al. Current status of enhanced recovery after surgery (ERAS) protocol in gastrointestinal surgery. Med Oncol 2018; 35: 95.

23. Powell R, Scott NW, Manyande A, et al. Psychological preparation and postoperative outcomes for adults undergoing surgery under general anaesthesia. Cochrane Database Syst Rev 2016; 2016: CD008646.

24. Bamgbade OA, Oluwole O, Khaw RR. Perioperative analgesia for fast-track laparoscopic bariatric surgery. Obes Surg 2017; 27: 1828-34.

25. Veiga-Gil L, Pueyo J, Lopez-Olaondo L. Postoperative nausea and vomiting: physiopathology, risk factors, prophylaxis and treatment. Rev Esp Anestesiol Reanim 2017; 64: 223-32. 
26. Mazzotta E, Villalobos-Hernandez EC, Fiorda-Diaz J, et al. Postoperative ileus and postoperative gastrointestinal tract dysfunction: pathogenic mechanisms and novel treatment strategies beyond colorectal Enhanced Recovery After Surgery Protocols. Front Pharmacol 2020; 11: 583422.

27. Cuthbertson D, Tilstone WJ. Metabolism during the postinjury period. Adv Clin Chem 1969; 12: 1-55.

28. Fink-Neuboeck N, Lindenmann J, Bajric S, et al. Clinical impact of interleukin 6 as a predictive biomarker in the early diagnosis of postoperative systemic inflammatory response syndrome after major thoracic surgery: a prospective clinical trial. Surgery 2016; 160: 443-53.

29. Tanaka T, Narazaki M, Kishimoto T. Interleukin (IL-6) Immunotherapy. Cold Spring Harb Perspect Biol 2018; 10:a028456.

30. Kanamori M, Nakatsukasa H, Okada M, et al. Induced regulatory T cells: their development, stability, and applications. Trends Immunol 2016; 37: 803-11.

31. Zhe C, Jian-wei L, Jian C, et al. Laparoscopic versus open splenectomy and esophagogastric devascularization for bleeding varices or severe hypersplenism: a comparative study. J Gastrointest Surg 2013; 17: 654-9.

32. Intagliata NM, Caldwell SH, Tripodi A. Diagnosis, development, and treatment of portal vein thrombosis in patients with and without cirrhosis. Gastroenterology 2019; 156: 1582-99.

33. Noronha FC, Reis D, Cortez-Pinto H, et al. Anticoagulation in cirrhosis and portal vein thrombosis is safe and improves prognosis in advanced cirrhosis. Dig Dis Sci 2019; 64: 2671-83.

34. Loffredo L, Pastori D, Farcomeni A, et al. Effects of anticoagulants in patients with cirrhosis and portal vein thrombosis: a systematic review and meta-analysis. Gastroenterology 2017 153: 480-7.

35. Glassou EN, Hansen TB, Pedersen AB. Risk of pneumonia and urinary tract infection within the first week after total hip arthroplasty and the impact on survival. Clin Epidemiol 2017; 9: 31-9.

36. Grant MC, Yang D, Wu CL, et al. Impact of enhanced recovery after surgery and fast track surgery pathways on healthcare-associated infections: results from a systematic review and meta-analysis. Ann Surg 2017; 265: 68-79.

Received: 10.08.2021, accepted: 12.11.2021. 